\title{
SARS coronavirus entry into host cells through a novel clathrin- and caveolae-independent endocytic pathway
}

\author{
Hongliang Wang ${ }^{1}$, Peng Yang ${ }^{1}$, Kangtai Liu ${ }^{1}$, Feng Guo ${ }^{1}$, Yanli Zhang ${ }^{1}$, Gongyi Zhang ${ }^{2}$, Chengyu Jiang ${ }^{1}$ \\ ${ }^{1}$ National Key Laboratory of Medical Molecular Biology, Institute of Basic Medical Sciences, Peking Union Medical College, Tsin- \\ ghua University and Chinese Academy of Medical Sciences, Beijing 100005, China; ${ }^{2}$ Department of Immunology, National Jewish \\ Medical and Research Center, Denver, CO 80206, USA
}

While severe acute respiratory syndrome coronavirus (SARS-CoV) was initially thought to enter cells through direct fusion with the plasma membrane, more recent evidence suggests that virus entry may also involve endocytosis. We have found that SARS-CoV enters cells via pH- and receptor-dependent endocytosis. Treatment of cells with either SARS-CoV spike protein or spike-bearing pseudoviruses resulted in the translocation of angiotensin-converting enzyme 2 (ACE2), the functional receptor of SARS-CoV, from the cell surface to endosomes. In addition, the spike-bearing pseudoviruses and early endosome antigen 1 were found to colocalize in endosomes. Further analyses using specific endocytic pathway inhibitors and dominant-negative Eps15 as well as caveolin-1 colocalization study suggested that virus entry was mediated by a clathrin- and caveolae-independent mechanism. Moreover, cholesterol- and sphingolipid-rich lipid raft microdomains in the plasma membrane, which have been shown to act as platforms for many physiological signaling pathways, were shown to be involved in virus entry. Endocytic entry of SARS-CoV may expand the cellular range of SARS-CoV infection, and our findings here contribute to the understanding of SARS-CoV pathogenesis, providing new information for anti-viral drug research.

Keywords: Severe Acute Respiratory Syndrome Coronavirus (SARS-CoV), endocytosis, angiotensin-converting enzyme 2 (ACE2), lipid rafts

Cell Research (2008) 18:290-301. doi: 10.1038/cr.2008.15; published online 29 January 2008

\section{Introduction}

Severe acute respiratory syndrome (SARS) emerged as an epidemic in Asia during the winter of 2002-2003 and quickly spread throughout the world. The etiologic agent was soon identified as a new coronavirus, SARS-CoV [1, 2]. The plague ended in July 2003, with a death toll of more than 700 patients and 8000 probable diagnoses [http:// www.who.int/csr/sars/country/table2004 04 21/en/index. $\mathrm{html}$. This disease had a significant impact on international social and economic activities. In addition to the likely

Correspondence: Chengyu Jiang

Tel: +86-10-65296908; Fax: +86-10-65276551

E-mail: jiang@pumc.edu.cn

Abbreviation: angiotensin converting enzyme 2 (ACE2); chlorpromazine (CPZ); cholera toxin subunit B (CTB); early endosome antigen 1 (EEA1); human embryonic kidney 293E (HEK293E); methyl- $\beta$-cyclodextrin (M $\beta C D)$; severe acute respiratory syndrome (SARS); severe acute respiratory syndrome coronavirus (SARS-CoV)

Received 27 November 2007; revised 29 November 2007; accepted 30 November 2007; published online 29 January 2008 reemergence of SARS, there are serious concerns about its potential misuse as a biological weapon. Four years after its identification, however, many pathological aspects of this virus have yet to be characterized.

The entry of enveloped viruses into cells is known to occur via two primary pathways: some viruses deliver their genomes to the cytosol after their envelopes fuse with the plasma membrane at the cell surface, whereas others take advantage of the cell's endocytic machinery. In the latter mechanism, the endocytosed virions are subjected to an activation step in the endosome, which is typically mediated by the acidic endosomal $\mathrm{pH}$, resulting in fusion of the viral and endosomal membranes and release of the viral genome into the cytosol. Therefore, the endocytic mechanism is thought to be a $\mathrm{pH}$-sensitive process, while direct membrane fusion is $\mathrm{pH}$-independent. The endocytic pathways exploited by animal viruses to gain entry into host cells include macropinocytosis, clathrin-dependent endocytosis, and caveolae-dependent endocytosis, as well as poorly characterized routes such as clathrin- and caveolae-independent endocytosis [3, 4]. Although most viruses 
use only one of these pathways to enter cells, recent studies have shown that some viruses use multiple mechanisms to gain entry into host cells [5-8].

The entry of SARS-CoV into cells was first identified to occur by direct fusion at the plasma membrane [9-11]. Some later studies have shown that entry of SARS-CoV may be $\mathrm{pH}$-dependent [12], and that the endosomal protease cathepsin L $[13,14]$ might be involved, suggesting that this virus may employ endocytosis. Because conflicting results have been obtained by various research groups using similar experimental methods, such as fluorospectrometric monitoring of GFP-labeled pseudovirus entry into host cells, we decided to use a different approach. We labeled the SARS-CoV functional receptor angiotensin converting enzyme 2 (ACE2) with GFP by stable transfection of human embryonic kidney 293E (HEK293E) cells. Receptor recycling was then tracked after the cells were treated with pseudoviruses or the spike protein, a membrane component of SARS-CoV that mediates membrane fusion and is required for viral entry $[11,12]$. This new approach greatly improves experimental stability and reliability. The results showed that SARS-CoV entered cells via receptor-dependent, $\mathrm{pH}$-sensitive endocytosis. We also showed that the specific endocytic pathway used by SARS-CoV to enter cells is clathrin- and caveolae-independent. Moreover, lipid rafts were found to play an important role in this process.
Our results provide further insight into the pathological characteristics of this newly emerged virus.

\section{Results}

Spike protein induced translocation of the SARS-CoV receptor ACE2 from the plasma membrane to cytoplasmic compartments

In order to examine the entry of SARS-CoV into cells, cell lines were first generated which stably expressed ACE2 fused to either a Myc tag or GFP. ACE2 expression in the 293E-ACE2-Myc cell lines was confirmed by western blotting (Supplementary information Figure S1), while ACE2 receptor expression in the 293E-ACE2-GFP cell lines was directly visualized by fluorescence. Figure $1 \mathrm{~A}$ shows that ACE2-GFP is localized primarily at the cell surface, suggesting that expression and localization of ACE2 in this cell line is similar to that in Vero E6 cells. The 293E-ACE2-GFP cell line allowed for direct tracking of the movement of the receptor under a fluorescence microscope. In agreement with a previous report, which showed that ACE2 expression in some nonpermissive cells renders them permissive to infection [15], both of these stable cell lines can be infected by spike-bearing pseudoviruses (Supplementary information Figure S1).

We first tested whether spike protein alone could enter
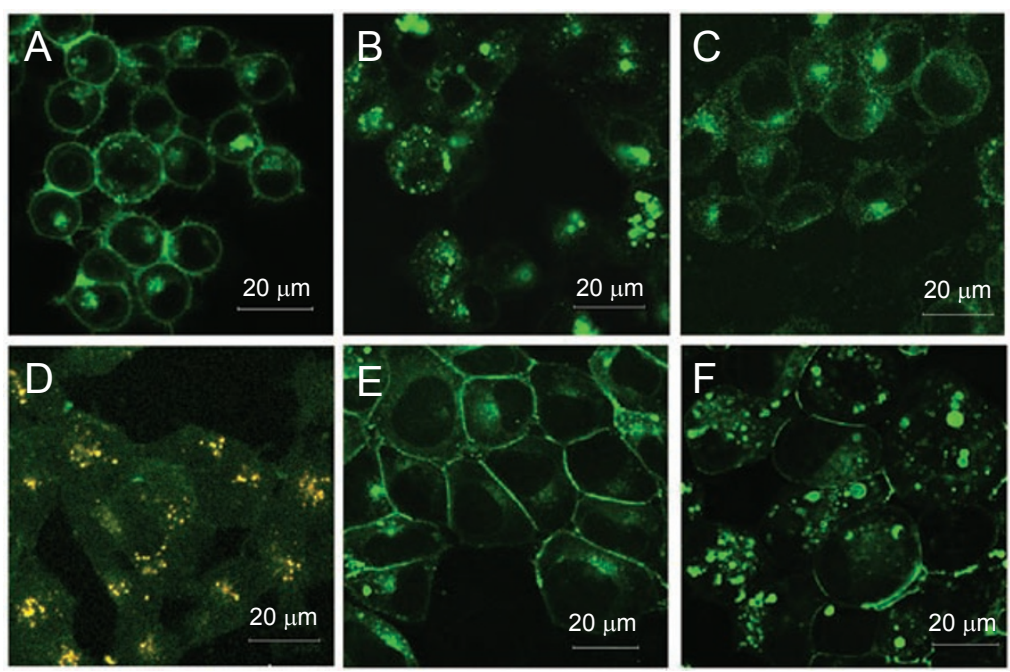

Figure 1 SARS-CoV receptor ACE2 translocates from the plasma membrane to cytoplasmic compartments following treatment with spike protein. (A) In HEK293E-ACE2-GFP cells, ACE2 is primarily located on the cell surface. (B) When HEK293EACE2-GFP cells were treated with the $\mathrm{S} 1190-\mathrm{Fc}$ protein (for $3 \mathrm{~h}$ at $37^{\circ} \mathrm{C}$ ), ACE2-GFP was internalized from the cell surface. (C) When HEK293E-ACE2-GFP cells were treated with Fc protein alone (for $3 \mathrm{~h}$ at $37^{\circ} \mathrm{C}$ ), no translocation of the SARS-CoV receptor ACE2 was observed. (D) Spike protein colocalizes with ACE2-GFP in cytoplasmic vesicles after $3 \mathrm{~h}$ incubation at 37 ${ }^{\circ} \mathrm{C}$. Spike-Fc protein was probed with Alexa-568 goat anti-human IgG. (E) ACE2 receptor recycling was observed after a 14-h incubation with spike-Fc at $37^{\circ} \mathrm{C}$; few green vesicles were visible in the cells after $14 \mathrm{~h}$. (F) HEK293E-ACE2-GFP cells were treated with $\mathrm{NH}_{4} \mathrm{Cl}$ before they were incubated with $\mathrm{S} 1190-\mathrm{Fc}$ protein. ACE2 was trapped in cytoplasmic vesicles, even after $14 \mathrm{~h}$. Scale bar: $20 \mu \mathrm{m}$. 
cells using the 293E-ACE2-GFP cell line, since spike protein was believed to mediate virus entry. Purified recombinant spike protein fused to the human IgG Fc fragment (S1190-Fc) was added to 293E-ACE2-GFP cells. After incubation at $37^{\circ} \mathrm{C}$ for $3 \mathrm{~h}$, a number of GFP-containing vesicles were observed in the perinuclear area (Figure 1B), whereas vesicles were not observed in the control cells treated with Fc protein alone (Figure 1C), suggesting that spike protein specifically induced translocation of ACE2GFP from the cell surface to the interior of the cells. Statistical analysis of the percentage of cells in which vesicles accumulated showed that there was a significant difference between the effects of these treatments on virus entry (Supplementary information Figure S2). A dual staining assay for spike protein and ACE2-GFP showed colocalization of these two proteins (Figure 1D), which suggests that spike protein was bound to ACE2 in the vesicles and that spike protein was taken up by the cell, most likely by endocytosis. The fact that spike protein induced translocation of ACE2 from the cell surface to intracellular compartments is in agreement with our previous finding that surface expression of ACE2 in Vero E6 cells decreased after incubation with spike protein at $37^{\circ} \mathrm{C}$ for $3 \mathrm{~h}$ [16].

After an additional incubation at $37^{\circ} \mathrm{C}$, receptor-containing vesicles were no longer visible within the cells, and green signals were instead seen clustered near the cell surface, suggesting that the receptors were recycled (Figure 1E). This process can be blocked by lysosomotropic agents, which are reported to function by elevating the $\mathrm{pH}$ of acidic compartments, thereby inhibiting dissociation of the ligand from the receptor and trapping the receptor in the endosome [17]. We found that after treatment with ammonium chloride, bafilomycin A1, or chloroquine, the viral receptor was trapped within perinuclear vacuoles, even after a 14-h incubation, a period that would allow the receptor to be recycled to the cell surface under normal conditions (Figure $1 \mathrm{~F}$ and unpublished data). These results reflect the normal traffic of membrane flow; that is, cargos are first internalized by a besieged membrane, which fuses with the early endosome and then the late endosome, where cargos are dissociated from the receptors. Receptors are usually recycled back to the cell membrane, while cargos are targeted to lysosomes.

\section{SARS-CoV spike-bearing pseudoviruses enter cells via} endocytosis

Due to the highly contagious nature of SARS-CoV, we used spike protein-bearing pseudoviruses to study the virus entry route. Pseudoviruses are often used to mimic the entry of real viruses, such as hepatitis $\mathrm{C}$ virus [18, 19], Ebola virus, and Marburg virus [20], into host cells. This strategy is a powerful tool for studying early events in the life cycle of a virus. Therefore, we used retroviral pseudoviruses bearing the SARS-CoV spike protein to infect ACE2-GFP-expressing HEK293E cells. After a 3-h incubation at $37{ }^{\circ} \mathrm{C}$, GFP-containing vesicles were detected within the cells (Figure 2A), while there were few intracellular vesicles when the same cell lines were treated with a control pseudovirus bearing VSV-G protein on the surface (Figure 2B). Dual labeling of spike protein and ACE2 also showed colocalization (Figure 2C), which indicates that the pseudovirus may be contained in the vesicles. After an additional $10 \mathrm{~h}$ incubation, few vesicles were observed (Figure 2D) for both treatments. This result was similar to that observed following treatment with spike protein alone. Moreover, after treatment with ammonium chloride, bafilomycin A1, or chloroquine, GFP-containing vesicles were detected, even after a 12-h incubation (Figure $2 \mathrm{E}$ and unpublished data), suggesting that these reagents inhibited receptor recycling. These results were nearly identical to those obtained following spike protein treatment, which indicated that SARS-CoV may enter cells via endocytosis. Although the pseudovirus can express GFP, the green vesicles observed in these cells at this time point resulted from cellular ACE2-GFP rather than from virally expressed GFP, as expression from the viral GFP gene was not observed at $12 \mathrm{~h}$ after infection. This notion was verified with ACE2-Myc-expressing HEK293E cells, in which no GFP expression was observed at $12 \mathrm{~h}$ postinfection with the pseudovirus (Supplementary information Figure S3).

The endocytic pathway is usually thought to be $\mathrm{pH}-$ dependent. Therefore, if SARS-CoV can enter cells via endocytosis, lysosomotropic agents should inhibit virus infection. Vero E6 cells have been reported to be naturally permissive for SARS-CoV. We infected Vero E6 cells with spike-bearing pseudovirus in the presence or absence of lysosomotropic agents. Since the pseudovirus expresses GFP, infected cells can be distinguished from uninfected cells by viral GFP expression. Figure $2 \mathrm{~F}$ shows that pseudovirus infection of Vero E6 cells leads to viral GFP expression, while the lysosomotropic agents inhibited GFP expression. This result suggests that successful virus entry is $\mathrm{pH}$-dependent.

To provide further confirmation that SARS-CoV enters host cells via endocytic pathways, dual immunofluorescence labeling with antibodies specific for the SARS-CoV spike protein and the early endosome marker protein early endosome antigen 1 (EEA1) was performed, followed by confocal microscopy analysis. After $1 \mathrm{~h}$ of infection, a punctate pattern characterized by strong colocalization of the spike protein and EEA1 was observed, confirming that SARS-CoV was targeted to early endosomes (Figure 2J-L). Alexa-labeled transferrin was used as a positive control (Figure 2G-I), since the endocytosis of transferrin has been 

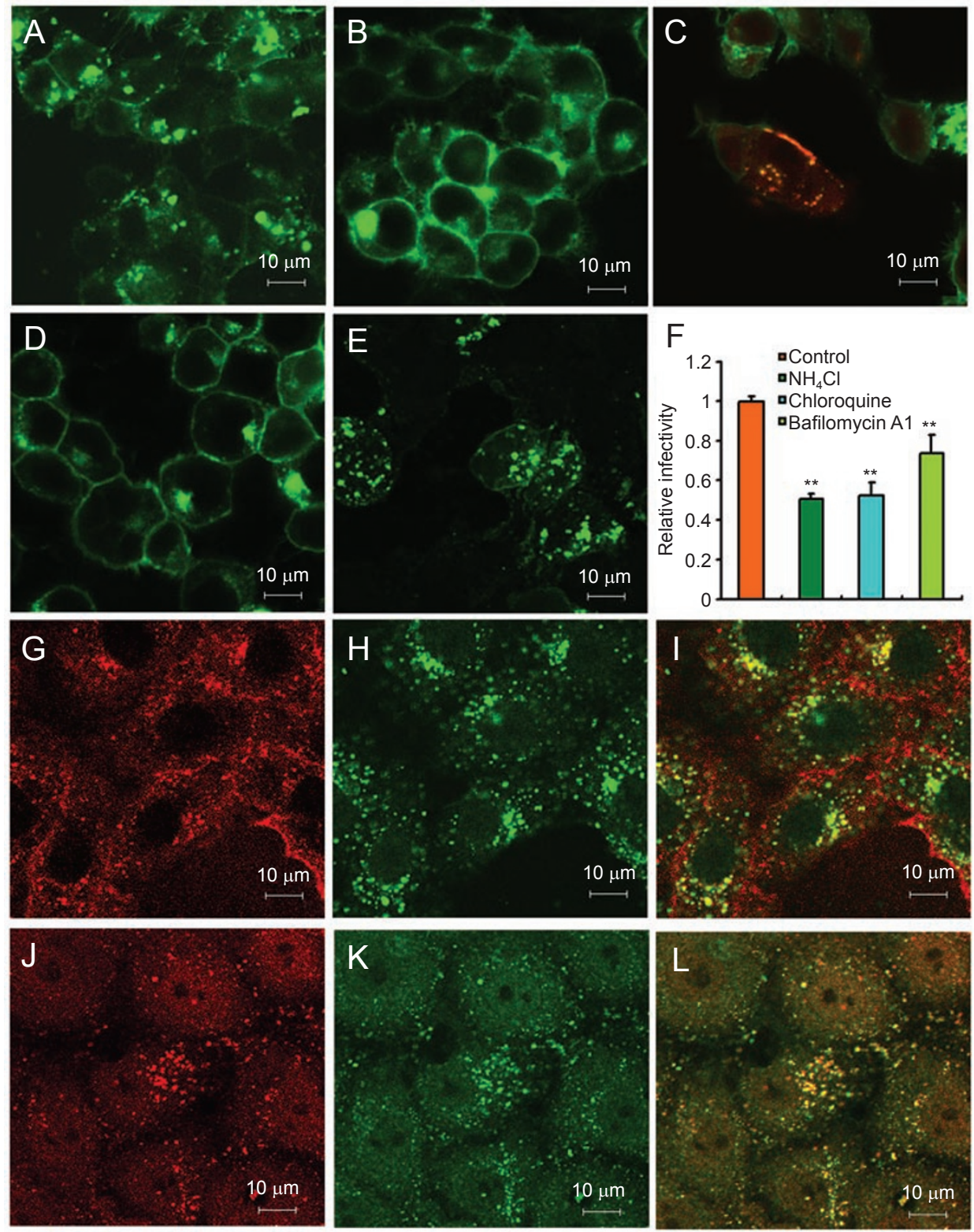

Figure 2 SARS-CoV spike-bearing pseudoviruses can enter cells via endocytosis. (A) When HEK293E-ACE2-GFP cells were treated with spike-bearing pseudovirus for $3 \mathrm{~h}$, translocation of the ACE2 receptor was observed. (B) When HEK293E-ACE2GFP cells were treated with spike minus pseudovirus (VSV-G pseudovirus) for $3 \mathrm{~h}$, no translocation of the ACE2 receptor was observed. (C) Colocalization of SARS-CoV spike protein and ACE2-GFP in cytoplasmic vesicles ( $3 \mathrm{~h}$ after treatment with spike-bearing pseudovirus). Spike protein was probed with primary antibody and then detected with Alexa-568 goat anti-mouse secondary antibody. (D) Twelve hours after treatment with spike-bearing pseudovirus, HEK293E-ACE2-GFP cells showed few cytoplasmic vesicles. (E) After chloroquine treatment, cytoplasmic vesicles were detected, even at $12 \mathrm{~h}$ after spike-bearing pseudovirus infection. (F) $\mathrm{NH}_{4} \mathrm{Cl}$, chloroquine, and bafilomycin $\mathrm{A} 1$ treatments inhibit viral GFP expression. Vero E6 cells were mock treated or pretreated with $50 \mathrm{mM} \mathrm{NH}_{4} \mathrm{Cl}, 100 \mu \mathrm{M}$ chloroquine, or $80 \mathrm{nM}$ bafilomycin $\mathrm{A} 1$ for $1 \mathrm{~h}$ before pseudovirus infection. After $48 \mathrm{~h}$, the cells were lysed and GFP was measured as described in the Materials and Methods section. Double asterisks indicate a significant difference from controls $(P<0.01, t$-test). Error bars represent the SD of three independent experiments. (G-I) Alexa 594 transferrin (G) and EEA1 (H) colocalized in Vero E6 cells after a 1-h incubation at $37^{\circ} \mathrm{C}$. EEA1 was detected with its specific antibody followed by Alexa 488 secondary antibody. (J-L) Vero E6 cells were infected with spike-bearing pseudovirus for $1 \mathrm{~h}$ before they were fixed and immunolabeled with primary antibodies specific for SARS-CoV spike protein (J) and the early endosome marker EEA1 (K). These two proteins were found to colocalize. Spike protein was detected by Alexa 568 secondary antibody, while EEA1 was detected with Alexa 488 secondary antibody. 
well characterized. After binding of transferrin to its receptor, the complex was shown to be internalized in coated vesicles, followed by fusion with endosomes [21-23].

Thus, the spike-bearing pseudovirus, like spike protein, can cause ACE2 receptor translocation in a $\mathrm{pH}$-dependent manner, and the pseudovirus infects Vero E6 cells in a pHdependent manner. In addition, the pseudovirus is targeted to the early endosome after it enters the cell. Taken together, these results indicate that the spike-bearing pseudovirus can enter cells via endocytosis.

\section{SARS-CoV can enter cells in the absence of clathrin-medi- ated endocytosis}

After determining that SARS-CoV can enter cells via endocytosis, we attempted to identify the specific endocytic pathway exploited by this virus. The endocytic pathways exploited by animal viruses to enter host cells include macropinocytosis, the clathrin-dependent pathway, and the caveolae-dependent pathway, as well as routes that are not as well characterized, such as clathrin- and caveolae-independent pathways. The clathrin-dependent pathway is the most common of these pathways. Because drugs can produce pleiotropic effects, we employed several complementary approaches to determine the role of clathrin-mediated endocytosis in the entry of SARS-CoV into cells [4].

Chlorpromazine (CPZ), a drug commonly used to inhibit clathrin-mediated endocytosis, causes clathrin lattices to assemble on endosomal membranes and prevents the assembly of coated pits at the cell surface [24]. The effect of $\mathrm{CPZ}$ on clathrin-mediated endocytosis was first tested with transferrin labeled with Alexa 594 (Alexa 594 Tf) using Vero E6 cells. In mock-treated cells, transferrin appeared to cluster in the perinuclear region, while in the CPZ-treated cells, transferrin uptake was blocked, leaving transferrin at the cell surface (Figure 3A and 3B). This result confirms the effectiveness of CPZ. We then tested the effect of CPZ on spike-pseudovirus entry using two different methods: confocal microscopy and spectrofluorometer measurement. After Vero E6 cells were treated with CPZ and incubated with spike-bearing pseudoviruses for $1 \mathrm{~h}$, the ability of spike-bearing pseudovirus to enter Vero E6 cells was examined using confocal microscopy. Figure $3 \mathrm{C}$ and $3 \mathrm{D}$ showed that the spike-bearing pseudovirus can enter Vero E6 cells despite CPZ treatment. In order to quantify the effect of $\mathrm{CPZ}$ on viral entry, Vero E6 cells were treated with the indicated amounts of $\mathrm{CPZ}$ and were then infected with spike-bearing pseudoviruses. The relative infectivity of the viruses was determined by measuring the level of GFP expression at $60 \mathrm{~h}$ postinfection using a spectrofluorometer. Our results demonstrated that CPZ did not significantly inhibit virus entry (Figure 3E).
We then used the more specific siRNA approach to further explore the role of clathrin-mediated endocytosis in SARS-CoV entry. HEK293E-ACE2-Myc cells were used because of their high transfection efficiency. Cells were
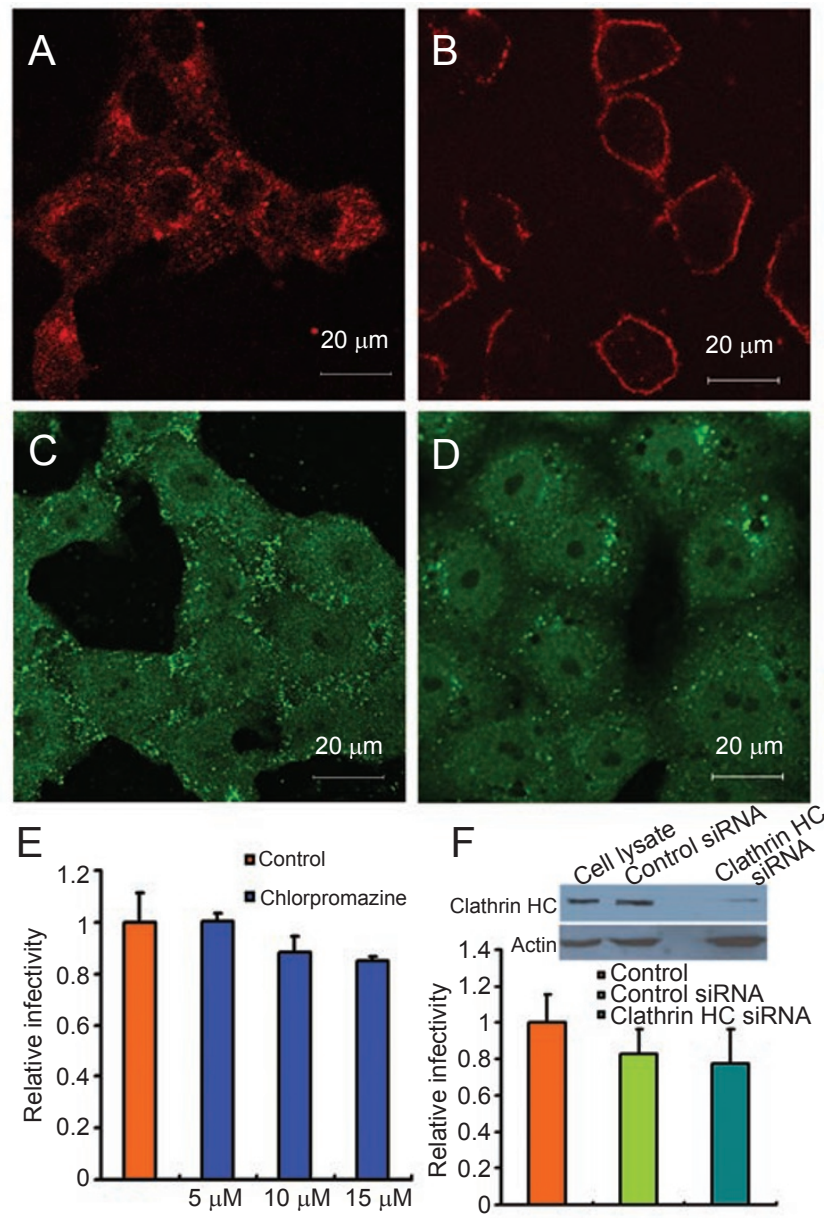

Figure 3 The effect of $\mathrm{CPZ}$ treatment and siRNA knockdown of clathrin on SARS-CoV entry. Vero E6 cells were mock treated (A and $\mathbf{C})$ or pretreated with CPZ $(10 \mu \mathrm{M})(\mathbf{B}$ and $\mathbf{D})$, and then incubated with Alexa 594 transferrin (A and B) or spike-bearing pseudovirus (C and $\mathbf{D}$ ) for $1 \mathrm{~h}$ at $37^{\circ} \mathrm{C}$. Spike protein was detected with anti-spike antibody, followed by Alexa 488 secondary antibody. (E) Vero E6 cells were treated with the indicated amount of chlorpromazine (CPZ) before they were incubated with GFPspike-bearing pseudovirus. Virus infectivity was measured using a spectrofluorometer at $60 \mathrm{~h}$ post-infection (see details in Materials and methods). Virus entry was not inhibited by this treatment. (F) HEK293 cells engineered to express ACE2-Myc were treated with siRNA specific for clathrin HC. Western blotting revealed that the siRNA markedly reduced the level of clathrin $\mathrm{HC}$. Cells were then treated with GFP-spike-bearing pseudoviruses, and the infectivity was measured using a spectrofluorometer at $48 \mathrm{~h}$ post-infection. The siRNA treatment did not inhibit virus entry. Error bars represent the SD of three independent experiments. 
transfected with siRNA specific for clathrin heavy chain (HC) or control siRNA. Seventy-two hours post-transfection, the effects of the siRNA were assayed using western blotting and the cells were infected with pseudoviruses. After another $48 \mathrm{~h}$, the cells were lysed and viral GFP expression was quantified using a spectrofluorometer. Although the expression of clathrin $\mathrm{HC}$ was knocked-down in the cells transfected with the specific siRNA compared to cells transfected with control siRNA, the infectivity of the virus was not significantly affected (Figure 3F).

We further employed a dominant-negative variant of Eps 15 to explore the role of clathrin-mediated endocytosis in SARS-CoV entry. Eps 15 plays an important role in clathrin-mediated endocytosis, and expression of the dominantnegative Eps 15 construct GFP-E $\Delta 95 / 295$ has been reported to inhibit clathrin-mediated endocytosis, while the construct GFP-D3 $\Delta 2$ can be used as a control $[25,26]$. To confirm disruption of the clathrin-dependent endocytic pathway, the transfected cells were incubated with Alexa-594 transferrin. As expected, the E $\Delta 95 / 295$ construct, but not the D $3 \Delta 2$ construct, successfully inhibited transferrin uptake (Figure 4A-F). However, we found that SARS-CoV was able to enter cells expressing GFP-E $\Delta 95 / 295$ as efficiently as it entered cells expressing GFP-D3 $\Delta 2$ (Figure 4G-L), demonstrating that expression of the dominant-negative variant Eps 15 did not block SARS-CoV entry. All of these results indicate that SARS-CoV is able to enter cells that lack a functional clathrin-dependent endocytic pathway.

\section{SARS-CoV enters cells independent of caveolae-mediated endocytosis}

Caveolae-dependent endocytosis is a newly characterized endocytosis pathway. Caveolae are small, flask-shaped invaginations in the plasma membrane composed of high levels of cholesterol and glycosphingolipids as well as the integral membrane protein caveolin [27]. Because cholesterol is a prominent component of lipid rafts, which are involved in caveolae formation, sequestration of cholesterol with the sterol-binding drugs filipin and nystatin impairs caveolae-mediated endocytosis [4]. To determine whether SARS-CoV enters cells through a caveolae-mediated pathway, we treated cells with filipin and nystatin. As a control, the effects of these drugs on caveolae-mediated endocytosis were examined by measuring the uptake of cholera toxin subunit B (CTB), since it is targeted to caveolae and its uptake can be blocked by these kinds of drugs $[28,29]$. Data presented in Figure 5A-C indicate that these drugs block CTB uptake, since Alexa-594 CTB clustered at the cell surface following drug treatment, compared to its concentration near the nucleus in mock-treated cells (Supplementary information Figure S4). When treated cells were infected with pseudovirus, virus entry was not inhibited by treatment with filipin or nystatin (Figure 5D and $5 \mathrm{~F}$ ), but was inhibited by another drug, methyl- $\beta$-cyclodextrin $(\mathrm{M} \beta \mathrm{CD})$ (Figure 5E), an oligosaccharide used to deplete cholesterol from cell membranes. The effects of these drugs on virus entry were measured quantitatively. Treatment of Vero E6 cells with M $\beta C D$ was shown to inhibit pseudovirus entry in a dose-dependent manner (Figure. $5 \mathrm{H}$ ), while filipin and nystatin had no inhibitory effect on virus entry (Figure 5G and 5I).

To further study whether the caveolae-dependent pathway was involved in the endocytosis of this virus, a colocalization assay of spike protein and caveolin-1 was performed. The infected cells were immunolabeled for SARS-CoV spike protein as a marker of the pseudoviruses and for caveolin-1 as a marker of the caveolae. No significant colocalization of the pseudovirus and caveolin-1 specific signals was observed (Figure 5J-5L). Taken together, these results indicate that SARS-CoV is able to enter cells in a caveolin-independent manner.

\section{Discussion}

Viruses are obligatory intracellular parasites; they must enter host cells before they can initiate their life cycle. The entry of enveloped viruses into cells is known to occur via two primary pathways. Some viruses deliver their genomes to the cytosol after their envelopes fuse with the plasma membrane at the cell surface, whereas others take advantage of the cell's endocytic machinery. Direct membrane fusion at the cell surface is $\mathrm{pH}$-independent, while entry via the endocytic pathway usually depends on the low $\mathrm{pH}$ of endocytic vesicles [30].

It was previously thought that a virus used either direct membrane fusion at the cell surface or endocytosis to enter cells. However, recent studies have found that this distinction is not absolute. For example, HIV was first thought to enter cells via direct membrane fusion, and in 2000, its use of the endocytic pathway was also reported [5]. This is not the only exception. Newcastle disease virus has also been reported to enter cells via direct fusion at the plasma membrane and via endocytic pathways [31].

Electron microscopy and virus entry experiments that demonstrated the $\mathrm{pH}$-independence of SARS-CoV infection led to the belief that this virus enters cells as a result of direct membrane fusion [9, 10]. Later studies, however, have suggested that the endocytosis pathway may also be involved in virus entry [12-14]. Our observations of ACE2 receptor translocation and recycling, $\mathrm{pH}$-dependent viral entry and early endosome location of the viral spike protein indicate that SARS-CoV pseudoviruses may enter cells via receptor-dependent, $\mathrm{pH}$-sensitive endocytosis.

The classical endocytosis pathway moves vesicles from 


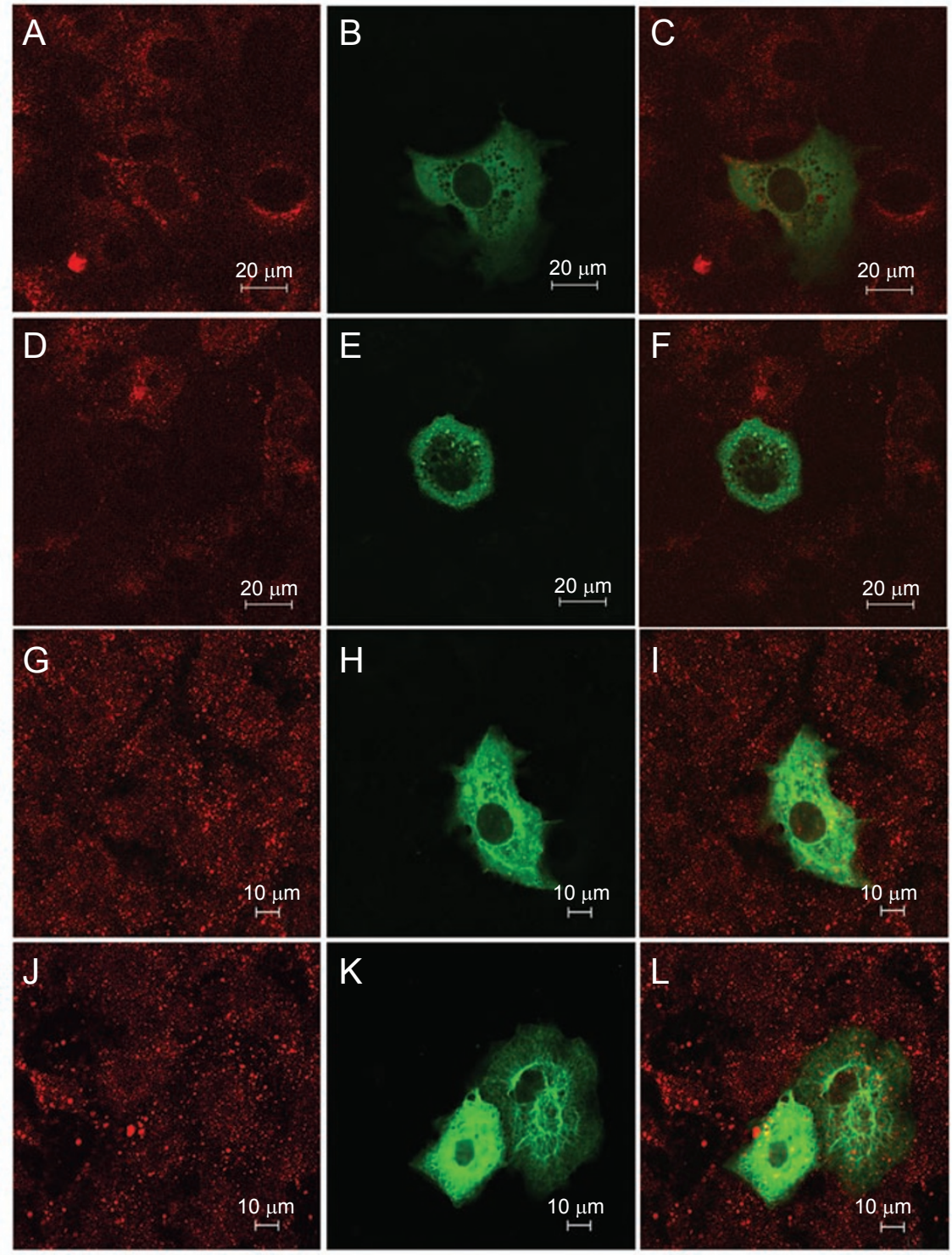

Figure 4 SARS-CoV entry into cells expressing a dominant-negative mutant of Eps15. Vero E6 cells were transfected with the GFP-tagged construct Eps15 D3 $\Delta 2$ (A-C and G-I) or E $\Delta$ 95/295 (D-F and J-L), 48 h later, cells were incubated with Alexa 594 transferrin (A-F) or infected with spike-bearing pseudovirus (G-L) for $1 \mathrm{~h}$ at $37^{\circ} \mathrm{C}$. Expression of Eps 15 proteins was monitored by GFP fluorescence. Spike-bearing pseudovirus was detected using anti-spike antibody, followed by incubation with Alexa-568 anti-mouse antibodies.

the early endosome to the late endosome, and then to the lysosome, where the internalized cargo is degraded by the acidic environment and various enzymes. Thus, it is possible that endocytic viral entry may lead to nonproductive infection because the viruses are degraded in the lysosome. In our experiments, however, we found that the viral genes were extensively expressed in all cell lines tested. The GFP protein encoded by the pseudovirus vector was first expressed at about $18 \mathrm{~h}$ postinfection (Supplementary information Figure S3). At this time point, virally produced GFP was evenly distributed in the cytoplasm of Vero E6 cells,
HEK293E-ACE2-Myc cells, and HEK293E-ACE2-GFP cells (as mentioned above, prior to infection, the majority of the GFP was localized on the cell surface of HEK239EACE2-GFP cells, Supplementary information Figure S1). Therefore, endocytic entry of SARS-CoV results in a productive infection.

Recent research has demonstrated that numerous viruses employ the endocytic machinery of the cell to infect cells. The endocytic pathways used include clathrin-mediated endocytosis, caveolae, macropinocytosis, and novel clathrin- and caveolae-independent pathways. Clathrin-medi- 

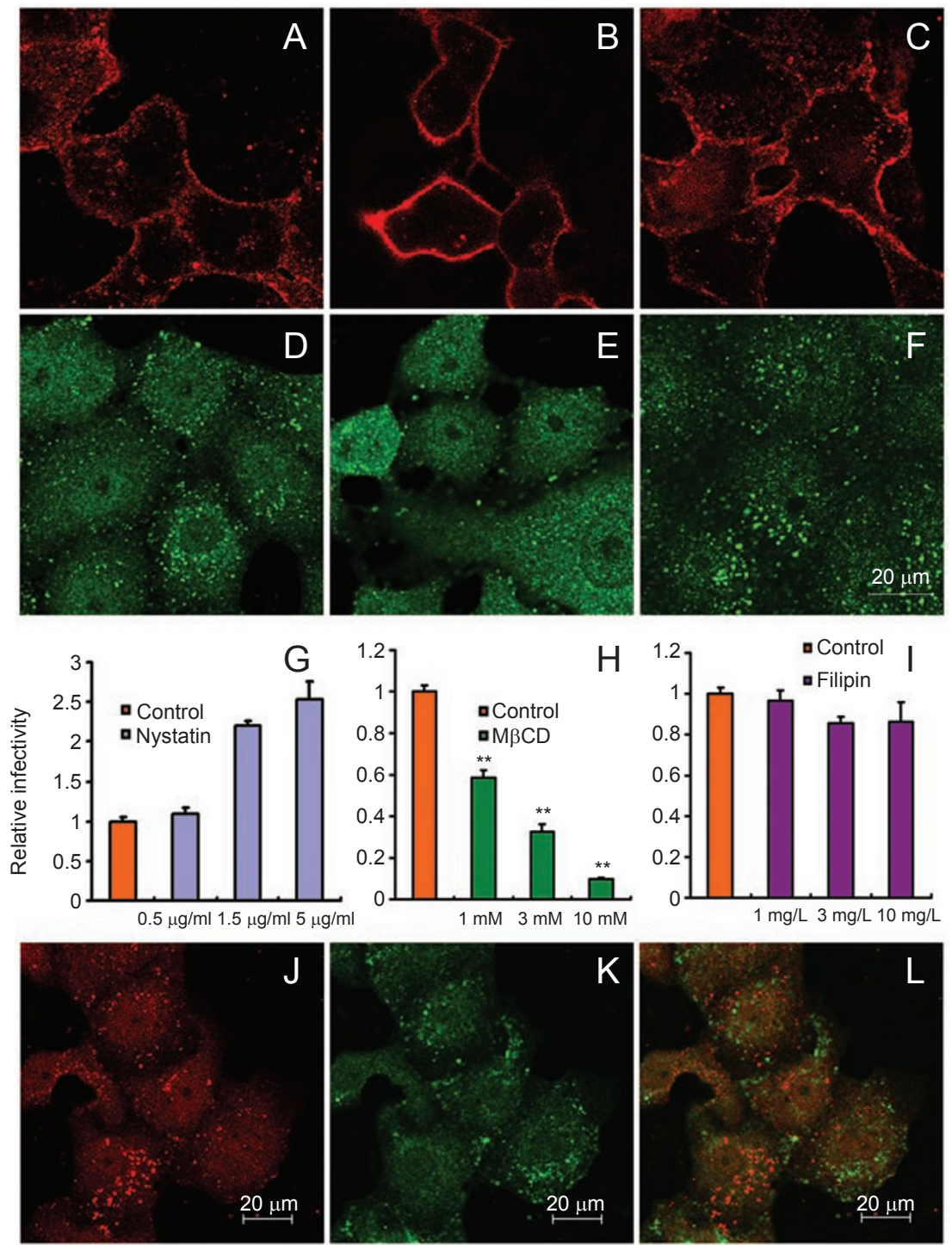

Figure 5 SARS-CoV entry into Vero E6 cells in the absence of caveolae-mediated endocytosis. Vero E6 cells were pretreated with nystatin (A, D, G), MBCD (B, E, H) or filipin(C, F, I), and were then incubated with Alexa-594-CTB (A-C) or spike-bearing pseudovirus (D-F and G-I). The spike-bearing pseudovirus in (D-F) was detected using anti-spike antibody (after $1 \mathrm{~h}$ incubation at $37^{\circ} \mathrm{C}$ ), followed by Alexa-488 anti-mouse antibodies, while in (G-I), pseudovirus was observed by monitoring GFP expression. Virus infectivity was measured using a spectrofluorometer at $60 \mathrm{~h}$ post-infection. (J-L) Colocalization of caveolin-1 (K) and spike-bearing pseudovirus $(\mathrm{J})$ was not observed (after $1 \mathrm{~h}$ incubation at $37^{\circ} \mathrm{C}$ ). Spike-bearing pseudovirus was detected using anti-spike antibody, followed by Alexa-568 anti-mouse antibodies, while caveolin-1 was detected using its specific primary antibody followed by Alexa-488 secondary antibody. Double asterisks indicate a significant difference from controls $(P<0.01$, t-test). Error bars represent the SD of three independent experiments.

ated endocytosis is the most common pathway. Numerous viruses use this pathway to enter cells, including Semliki forest virus (SFV), vesicular stomatitis virus (VSV), hepatitis $\mathrm{C}$ virus (HCV), and Adenoviruses 2 and 5 [3]. Therefore, we first determined whether SARS-CoV enters cells through clathrin-mediated endocytosis. Combined approaches of CPZ-mediated inhibition, clathrin heavy chain knockdown and dominant-negative Eps15 showed that SARS-CoV can infect cells in the absence of clathrinmediated endocytosis.

More recently, caveolae-mediated endocytosis was described. Caveolae are cholesterol- and sphingolipid-rich smooth invaginations of the plasma membrane which are generally associated with caveolin. SV40 and Ebola virus have been reported to use this pathway. Caveolae-mediated endocytosis can be disrupted by drugs that sequester 
cholesterol from the plasma membrane. In order to define the role of caveolae-mediated endocytosis in SARS-CoV entry, we used several drugs that affect this pathway, such as nystatin, filipin, and M $\beta C D$. In our experiments, neither nystatin nor filipin inhibited virus entry, while $\mathrm{M} \beta \mathrm{CD}$ was able to block virus entry in a dose-dependent manner, which was in agreement with a previous report by $\mathrm{Li}$ et al. [32]. These results, together with the fact that dual immunostaining showed that SARS-CoV and caveolin-1 did not colocalize, suggest that depletion of cholesterol by $\mathrm{M} \beta C D$ inhibits SARS-CoV entry, not through interfering with caveolae-mediated endocytosis per se but most likely by altering the integrity of membrane lipid microdomains. Therefore, these results suggest that caveolae-mediated endocytosis is not involved in the entry of SARS-CoV. This finding is in agreement with the fact that SARS-CoV can infect the colon carcinoma cell line Caco-2 [33, 34], which is devoid of caveolin-1 [35].

Finally, viruses can enter cells via macropinocytosis, a nonspecific internalization mechanism that does not require a receptor. Instead, endocytic vesicles form in response to cell stimulation, which causes lamellipodia at the sites of membrane ruffling to close and form large, irregular vesicles known as macropinosomes [4]. A wide variety of viruses have been reported to use macropinocytosis to gain entry into host cells, because macropinosomes can be acidified and intersect with endocytic vesicles [4]. The process of macropinocytosis requires actin; therefore, the most commonly used inhibitor of macropinocytosis is cytochalasin D. We found that treatment of Vero E6 cells with cytochalasin D slightly inhibited viral infection (Supplementary information Figure S5). However, macropinocytosis is unlikely to be the main pathway of entry for SARS-CoV, since SARS-CoV pseudoviruses induced receptor translocation from the cell surface to intracellular compartments, and macropinocytosis is not dependent on a receptor.

Most recently, another group reported that SARS-CoV may enter the hepatocellular carcinoma cell line HepG2 through clathrin-mediated endocytosis [36]. Importantly, clathrin-mediated endocytosis is primarily receptor-mediated, and previous research has shown that liver tissue is largely negative or low for ACE2 protein expression [37]. Although these authors also used engineered COS7 cells expressing ACE2, which otherwise lack endogenous ACE2 expression, these cell lines are atypical cell models in SARS-CoV studies. Since Vero E6 cells have a relatively high level of ACE2 expression and are widely used for SARS-CoV research, the precise pathway by which SARS-CoV enters Vero E6 cells needs to be elucidated. In our study, we used several complementary approached to investigate SARS-CoV entry in Vero E6 cells, including
CPZ inhibition, clathrin knockdown, and dominate-negative Eps15.

We have provided direct evidence that in addition to direct membrane fusion, SARS-CoV can enter cells through an endocytic route, and this endocytic infection leads to viral gene expression. Endocytic entry could expand the cellular range of SARS-CoV, and may explain the replication of SARS-CoV in ACE2-negative colonic enterocytes and liver tissue [37], because macropinocytosis-dependent entry does not require a specific receptor. Our results also suggest that SARS-CoV is able to enter cells through receptor-mediated, clathrin-independent, caveolae-independent endocytosis, likely through a process involving lipid rafts.

A previous report showed that cholesterol extraction by $\mathrm{M} \beta C D$ treatment slightly reduced the expression of ACE2 at the cell surface in Vero E6 cells. Importantly, there is no direct colocalization of ACE2 and lipid rafts [32]. This scenario is similar to that of the HIV-1 coreceptor CXCR4, which, despite preferential association with the nonraft membrane fraction of cells, requires cholesterol for normal function [38]. This observation can be explained by the complicated conformational changes that occur during virus entry. For example, in the case of HIV-1, after virus binding to CD4, the CD4-HIV-1 complex may either leave the raft compartment in order to associate with CXCR4 outside of rafts or stimulate entry of CXCR4 into rafts. A similar scenario may exist for SARS-CoV. Although ACE2 does not directly localize in rafts, some unknown factors involved in virus entry may localize to lipid rafts. Further detailed experiments are necessary to determine whether host factors other than ACE2 are involved in virus entry, and, if so, whether they localize to lipid rafts and how they interact with ACE2 to facilitate virus entry.

Lipid rafts are sphingolipid- and cholesterol-rich domains of plasma membrane that contain a variety of signaling and transport proteins. Lipid raft signaling has been reported to be important in the pathogenesis of many diseases [39], so these microdomains of plasma membrane have been pharmacologically targeted to prevent and treat these diseases. SARS-CoV is known to cause acute respiratory distress syndrome (ARDS), the leading cause of death due to SARS-CoV infection, as well as a hemophagocytic syndrome, lymphoid depletion, and skeletal muscle fiber necrosis, which all result from elevated levels of proinflammatory cytokines. Our previous studies showed that ARDS caused by SARS-CoV is mediated by the renin-angiotensin system and decreased levels of ACE2 protein [16] due to downregulation of $A C E 2$ transcription (unpublished data). The fact that endocytic entry of SARS-CoV involves lipid rafts provides new insight into ARDS pathogenesis and opens up new avenues which could elucidate the mo- 
lecular pathogenesis of elevated proinflammatory cytokine levels.

These findings are highly relevant for the development of future therapies directed at SARS-CoV. To date, there are no specific antiviral drugs that have been shown to effectively treat SARS. Characterization of the molecular pathogenesis of SARS-CoV and the evaluation of novel targets has led to the suggestion that entry inhibitors may prove to be valuable for the treatment of this disease [40, 41]. Our findings here provide additional information that can be used for drug design.

\section{Materials and Methods}

\section{Generation of cells lines stably expressing ACE2}

Peak 13 plasmids (provided by Dr Brian Seed) encoding human ACE2-Myc, human ACE2-GFP, or a control plasmid were transfected into HEK293E cells with Lipofectamine 2000 (Invitrogen). Puromycin-resistant clones with high levels of ACE2-Myc and ACE2-GFP expression were detected using western blotting and fluorescence microscopy, respectively.

\section{Expression and purification of spike protein and pseudo- virus production}

HEK293E cells stably expressing S1190-Fc were generated using the methods described above. The protein was purified with a protein A column (GE Healthcare), and the protein concentration was measured with a protein assay kit from Bio-Rad. Spike protein-bearing pseudotyped virus stocks were produced as previously reported [15]. Briefly, HEK293T cells were cotransfected with the pQCXIX vector, a spike-encoding plasmid (sh-2, provided by Dr Michael Farzan), or a control plasmid (VSV-G), and a gag/pol expression plasmid. The pQCXIX vector has been engineered to insert an EGFP gene. At $48 \mathrm{~h}$ post-transfection, virus supernatants were harvested and filtered through $0.45 \mu \mathrm{m}$ pore-size filters. Viral stocks were aliquoted and frozen at $-80^{\circ} \mathrm{C}$. Virus titers were determined as previously described [42].

Spike protein- or pseudovirus-induced receptor translocation

HEK293E-ACE2-GFP cells $\left(3 \times 10^{4}\right.$ cells $)$ were seeded onto plates. Twenty-four hours later, supernatant containing the spike-bearing pseudoviruses, the control pseudovirus, $100 \mu \mathrm{g}$ of S1190-Fc, or $25 \mu \mathrm{g}$ of $\mathrm{Fc}$ (equimolar amounts of the proteins) were added to the cells. After a 3-h incubation at $37^{\circ} \mathrm{C}$, the cells were observed under a fluorescence microscope. For the experiments using lysosomotropic reagents, cells were pretreated with $40 \mathrm{mM} \mathrm{NH}_{4} \mathrm{Cl}, 80 \mathrm{nM}$ bafilomycin A1, or $100 \mu \mathrm{M}$ chloroquine (Sigma) for $1 \mathrm{~h}$ at $37^{\circ} \mathrm{C}$. The cells were then treated for $3 \mathrm{~h}$ with protein solutions or with the pseudovirus supernatant containing the same reagent concentrations. Following this incubation, the cells were returned to solutions containing the reagents without the proteins or pseudoviruses. After 9-11 h, the cells were observed under a Leica fluorescence confocal microscope.

\section{Immunofluorescence labeling}

Vero E6 cells were grown on coverslips to approximately $80 \%$ confluence before they were spin-infected with pseudovirus. One hour postinfection, cells were fixed with $4 \%$ PFA, permeabilized with $0.1 \%$ Triton X-100, and blocked with $3 \%$ BSA. Cells were then probed with primary antibodies for $1 \mathrm{~h}$ (anti-caveolin-1 and anti-EEA1 antibodies from Santa Cruz Biotechnology, anti-spike antibodies produced as previously described [43]). After three washes with PBS, caveolin-1 was detected with Alexa Fluor 488 goat anti-rabbit antibodies (Molecular Probes), EEA1 was detected with Alexa Fluor 488 rabbit anti-goat antibodies (Molecular Probes), and spike protein was detected with Alexa Fluor 568 or 488 goat anti-mouse antibodies (Molecular Probes). Cells were washed three times with PBS and mounted on glass slides. For the dominant-negative protein assay, Vero E6 cells were transfected with plasmids encoding GFP-E $\Delta 95 / 295$ or GFP-D3 $\Delta 2$ (provided by Alexandre Benmerah through Dr Du Feng) with Lipofectamine 2000. Forty-eight hours later, cells were infected with pseudovirus and immunolabeled as described above. Pseudoviruses were immunolabeled with anti-spike antibodies and detected with Alexa Fluor 568 goat anti-mouse secondary antibodies. Primary and secondary antibodies were used at 1:200 and 1:400 dilutions, respectively. Images were obtained with a Leica confocal microscope.

\section{Confocal image acquisition and processing}

For the receptor translocation and immunolabeling experiments, confocal microscopy and sequential confocal analyses were performed using a Leica laser-scanning spectrum confocal system linked to a microscope (Leica TCS SP2). Specific PMT adjustments for GFP, 488 and 568 $\mathrm{nm}$, were used as appropriate. Images were captured using a $100 \times$ oil objective (Plan-Apo 1.4) at room temperature with the confocal acquisition software LCS (Leica). For some of the images, brightness and contrast adjustments were applied to the entire image using Adobe Photoshop 7.0.

\section{Drug inhibition of pseudovirus entry into Vero E6 cells}

Drug inhibition experiments were carried out as previously described [44, 45]. Briefly, Vero E6 cells were pretreated with the indicated amount of $\mathrm{CPZ}, \mathrm{M} \beta \mathrm{CD}$, nystatin, filipin, or cytochalasin D (Sigma) for $45 \mathrm{~min}$ at $37^{\circ} \mathrm{C}$ before 
infection. For the confocal microscopy analyses, treated cells were incubated with pseudovirus for $1 \mathrm{~h}$ at $37^{\circ} \mathrm{C}$ before they were fixed and probed with anti-spike primary antibody and Alexa-488 goat anti-mouse antibody (Molecular Probes), as described above. For the spectrofluorometry experiment, treated cells were infected with pseudovirus for $2 \mathrm{~h}$ at $37^{\circ} \mathrm{C}$ in the presence of drugs before fresh medium was added, and the cells were incubated at $37^{\circ} \mathrm{C}$ for $60 \mathrm{~h}$. Cells were lysed with lysis buffer (1\% NP-40, 150 $\mathrm{mM} \mathrm{NaCl}, 10 \mathrm{mM}$ Tris- $\mathrm{HCl} \mathrm{pH}$ 8.0, 0.02\% sodium azide, $2 \mathrm{mM}$ EDTA pH 8.0, and $100 \mu \mathrm{g} / \mathrm{ml}$ DTT) in the presence of $100 \mu \mathrm{g} / \mathrm{ml}$ PMSF protease inhibitor. After the cell debris was removed by centrifugation at $10000 \mathrm{rpm}$ for $10 \mathrm{~min}$ at $4{ }^{\circ} \mathrm{C}$, GFP was quantified with an LS-55 spectrofluorometer (Perkin-Elmer) with an excitation wavelength of $488 \mathrm{~nm}$ and an emission of $507 \mathrm{~nm}$. The data were further normalized to the total cell lysate protein concentration, which was measured with a Bio-Rad kit.

\section{SiRNA assay}

The siRNA assay was carried out as previously described [45]. Briefly, HEK293E-ACE2-Myc cells ( $10^{4}$ cells $)$ were transfected with siRNA directed against clathrin HC (80 pmol; Santa Cruz Biotechnology) or control siRNA using Lipofectamine 2000. Seventy-two hours later, the effect of the siRNA treatment was determined by western blotting using a clathrin $\mathrm{HC}$ antibody from Santa Cruz. In parallel, cells were infected with SARS pseudovirus, and GFP expression was examined $48 \mathrm{~h}$ later, as described above.

\section{Transferrin and CTB uptake assays}

Vero E6 cells were plated on coverslips the day before they were used. Cells were pretreated or transfected as described above and incubated with $5.0 \mu \mathrm{g}$ Alexa-594 Transferrin or Alexa-594 CTB (Molecular Probes) $/ \mathrm{ml}$ for 30 min at $4{ }^{\circ} \mathrm{C}$. The cells were then transferred to $37{ }^{\circ} \mathrm{C}$ for $1 \mathrm{~h}$. Un-internalized ligands were removed by washing with $0.1 \mathrm{M}$ glycine, $0.1 \mathrm{M} \mathrm{NaCl}$ at $\mathrm{pH}$ 3.0. The cells were then fixed and analyzed.

\section{Statistical analysis}

To compare S1190-Fc- and Fc-induced receptor translocation, cells containing obvious vesicles were counted as positive after a 3-h incubation. In order to eliminate the potential bias of the researchers, the cells were counted and analyzed by a college student from Shenyang Pharmaceutical University, Xiangwu Ju.

Related results are presented as the mean $\pm \mathrm{SD}$ from at least three independent experiments. Mean values were statistically compared using two-tailed Student's $t$-tests with a confidence level of $95 \%$.

\section{Acknowledgments}

We thank Dr James Kappler for editing. We thank Drs Brian Seed and Michael Farzan from Harvard Medical School, Dr Alexandre Benmerah ( through Dr Du Feng, Tsinghua University, China) from Institut Pasteur for providing materials and reagents. We thank Ju Xiangwu from Shenyang Pharmaceutical University, China for the statistic analysis. This work was funded by National Natural Science Foundation of China (30421003, 30528002, 30625013, and 30623009) and Ministry of Science and Technology of China (2006AA02Z152 and 2005CB523000). Y. Z. was supported by NNSFC 30600020.

\section{References}

1 Drosten C, Gunther S, Preiser W, et al. Identification of a novel coronavirus in patients with severe acute respiratory syndrome. N Engl J Med 2003; 348:1967-1976.

2 Ksiazek TG, Erdman D, Goldsmith CS, et al. A novel coronavirus associated with severe acute respiratory syndrome. $N$ Engl J Med 2003; 348:1953-1966.

3 Pelkmans L, Helenius A. Insider information: what viruses tell us about endocytosis. Curr Opin Cell Biol 2003; 15:414-422.

4 Sieczkarski SB, Whittaker GR. Dissecting virus entry via endocytosis. J Gen Virol 2002; 83:1535-1545.

5 Fackler OT, Peterlin BM. Endocytic entry of HIV-1. Curr Biol 2000; 10:1005-1008.

6 Matlin KS, Reggio H, Helenius A, Simons K. Infectious entry pathway of influenza virus in a canine kidney cell line. $J$ Cell Biol 1981; 91:601-613.

7 Sieczkarski SB, Whittaker GR. Influenza virus can enter and infect cells in the absence of clathrin-mediated endocytosis. $J$ Virol 2002; 76:10455-10464.

8 Nunes-Correia I, Eulalio A, Nir S, Pedroso de Lima MC. Caveolae as an additional route for influenza virus endocytosis in MDCK cells. Cell Mol Biol Lett 2004; 9:47-60.

$9 \mathrm{Ng}$ ML, Tan SH, See EE, Ooi EE, Ling AE. Early events of SARS coronavirus infection in vero cells. J Med Virol 2003; 71:323-331.

10 Qinfen Z, Jinming C, Xiaojun H, et al. The life cycle of SARS coronavirus in Vero E6 cells. J Med Virol 2004; 73:332-337.

11 Simmons G, Reeves JD, Rennekamp AJ, Amberg SM, Piefer AJ, Bates P. Characterization of severe acute respiratory syndromeassociated coronavirus (SARS-CoV) spike glycoprotein-mediated viral entry. Proc Natl Acad Sci USA 2004; 101:4240-4245.

12 Yang ZY, Huang Y, Ganesh L, et al. pH-dependent entry of severe acute respiratory syndrome coronavirus is mediated by the spike glycoprotein and enhanced by dendritic cell transfer through DC-SIGN. $J$ Virol 2004; 78:5642-5650.

13 Huang IC, Bosch BJ, Li F, et al. SARS coronavirus, but not human coronavirus NL63, utilizes cathepsin L to infect ACE2expressing cells. J Biol Chem 2006; 281:3198-3203.

14 Simmons G, Gosalia DN, Rennekamp AJ, Reeves JD, Diamond SL, Bates P. Inhibitors of cathepsin L prevent severe acute respiratory syndrome coronavirus entry. Proc Natl Acad Sci USA 2005; 102:11876-11881. 
15 Moore MJ, Dorfman T, Li W, et al. Retroviruses pseudotyped with the severe acute respiratory syndrome coronavirus spike protein efficiently infect cells expressing angiotensin-converting enzyme 2. J Virol 2004; 78:10628-10635.

16 Kuba K, Imai Y, Rao S, et al. A crucial role of angiotensin converting enzyme 2 (ACE2) in SARS coronavirus-induced lung injury. Nat Med 2005; 11:875-879.

17 Ray RB, Basu A, Steele R, et al. Ebola virus glycoprotein-mediated anoikis of primary human cardiac microvascular endothelial cells. Virology 2004; 321:181-188.

18 Blanchard E, Belouzard S, Goueslain L, et al. Hepatitis C virus entry depends on clathrin-mediated endocytosis. J Virol 2006; 80:6964-6972.

19 Codran A, Royer C, Jaeck D, et al. Entry of hepatitis C virus pseudotypes into primary human hepatocytes by clathrin-dependent endocytosis. J Gen Virol 2006; 87:2583-2593.

20 Empig CJ, Goldsmith MA. Association of the caveola vesicular system with cellular entry by filoviruses. J Virol 2002; 76:52665270.

21 Dautry-Varsat A. Receptor-mediated endocytosis: the intracellular journey of transferrin and its receptor. Biochimie 1986; 68:375-381.

22 Dautry-Varsat A, Ciechanover A, Lodish HF. $\mathrm{pH}$ and the recycling of transferrin during receptor-mediated endocytosis. Proc Natl Acad Sci USA 1983; 80:2258-2262.

23 Harding C, Heuser J, Stahl P. Receptor-mediated endocytosis of transferrin and recycling of the transferrin receptor in rat reticulocytes. J Cell Biol 1983; 97:329-339.

24 Wang LH, Rothberg KG, Anderson RG. Mis-assembly of clathrin lattices on endosomes reveals a regulatory switch for coated pit formation. J Cell Biol 1993; 123:1107-1117.

25 Benmerah A, Bayrou M, Cerf-Bensussan N, Dautry-Varsat A. Inhibition of clathrin-coated pit assembly by an Eps 15 mutant. J Cell Sci 1999; 112 Part 9:1303-1311.

26 Benmerah A, Poupon V, Cerf-Bensussan N, Dautry-Varsat A. Mapping of Eps15 domains involved in its targeting to clathrincoated pits. J Biol Chem 2000; 275:3288-3295.

27 Stan RV. Structure of caveolae. Biochim Biophys Acta 2005; 1746:334-348.

28 Orlandi PA, Fishman PH. Filipin-dependent inhibition of cholera toxin: evidence for toxin internalization and activation through caveolae-like domains. J Cell Biol 1998; 141:905-915.

29 Sanchez-San Martin C, Lopez T, Arias CF, Lopez S. Characterization of rotavirus cell entry. J Virol 2004; 78:2310-2318.

30 Lanzrein M, Schlegel A, Kempf C. Entry and uncoating of enveloped viruses. Biochem J 1994; 302 (Part 2):313-320.

31 Cantin C, Holguera J, Ferreira L, Villar E, Munoz-Barroso I.
Newcastle disease virus may enter cells by caveolae-mediated endocytosis. J Gen Virol 2007; 88:559-569.

32 Li GM, Li YG, Yamate M, Li SM, Ikuta K. Lipid rafts play an important role in the early stage of severe acute respiratory syndrome-coronavirus life cycle. Microbes Infect 2007; 9:96-102.

33 Cinatl J Jr, Hoever G, Morgenstern B, et al. Infection of cultured intestinal epithelial cells with severe acute respiratory syndrome coronavirus. Cell Mol Life Sci 2004; 61:2100-2112.

34 Mossel EC, Huang C, Narayanan K, Makino S, Tesh RB, Peters CJ. Exogenous ACE2 expression allows refractory cell lines to support severe acute respiratory syndrome coronavirus replication. J Virol 2005; 79:3846-3850.

35 Mirre C, Monlauzeur L, Garcia M, Delgrossi MH, Le Bivic A. Detergent-resistant membrane microdomains from Caco-2 cells do not contain caveolin. Am J Physiol 1996; 271:C887-C894.

36 Inoue Y, Tanaka N, Tanaka Y, et al. Clathrin-dependent entry of SARS coronavirus into target cells expressing cytoplasmic tail-deleted ACE2. J Virol 2007; 81:8722-8729.

37 Hamming I, Timens W, Bulthuis ML, Lely AT, Navis GJ, van Goor H. Tissue distribution of ACE2 protein, the functional receptor for SARS coronavirus. A first step in understanding SARS pathogenesis. J Pathol 2004; 203:631-637.

38 Popik W, Alce TM, Au WC. Human immunodeficiency virus type 1 uses lipid raft-colocalized CD4 and chemokine receptors for productive entry into CD4(+) T cells. J Virol 2002; 76:47094722.

39 Manes S, del Real G, Martinez AC. Pathogens: raft hijackers. Nat Rev 2003; 3:557-568.

40 Shigeta S, Yamase T. Current status of anti-SARS agents. Antivir Chem Chemother 2005; 16:23-31.

41 Kliger Y, Levanon EY, Gerber D. From genome to antivirals: SARS as a test tube. Drug Disc Today 2005; 10:345-352.

42 Negre D, Mangeot PE, Duisit G, et al. Characterization of novel safe lentiviral vectors derived from simian immunodeficiency virus (SIVmac251) that efficiently transduce mature human dendritic cells. Gene Ther 2000; 7:1613-1623.

43 Kohler G, Milstein C. Continuous cultures of fused cells secreting antibody of predefined specificity. Nature 1975; 256:495-497.

44 Kee SH, Cho EJ, Song JW, Park KS, Baek LJ, Song KJ. Effects of endocytosis inhibitory drugs on rubella virus entry into VeroE6 cells. Microbiol Immunol 2004; 48:823-829.

45 Meertens L, Bertaux C, Dragic T. Hepatitis C virus entry requires a critical postinternalization step and delivery to early endosomes via clathrin-coated vesicles. J Virol 2006; 80:11571-11578.

(Supplementary Information is linked to the online version of the paper on the Cell Research website.) 